\title{
On the Computation of Electric Fields from a Lightning Discharge in Time Domain
}

\author{
Rajeev Thottappillil \\ Ångström Laboratory, Uppsala University \\ Box 539 \\ 75121 Uppsala, Sweden
}

\author{
Vladimir A. Rakov \\ Department of Electrical and Computer Engineering \\ University of Florida, Gainesville \\ FL-32611, USA
}

\begin{abstract}
Two different approaches to the computation of electric fields from the lightning return stroke in the time domain are compared and discussed. These approaches are the dipole technique and the monopole technique. Lightning channel is modeled as a linear antenna. It is shown analytically for the case of an arbitrary source distribution on the antenna that both these techniques yield the same total fields and same Poynting vectors and therefore are equivalent. However, the expressions for the individual field components in time domain for these techniques, traditionally identified by their distance dependence as electrostatic, induction, and radiation terms, are different. The differences between the corresponding field components are considerable at close ranges, but become negligible at far ranges.
\end{abstract}

\section{INTRODUCTION}

Rubinstein and Uman [1] discussed two equivalent approaches to calculating the electric fields produced by a specified source. The first approach, the so-called dipole technique, involves

(1) the specification of current density $\bar{J}$;

(2) the use of $\bar{J}$ to find the vector potential $\bar{A}$;

(3) the use of $\bar{A}$ and the Lorentz condition to find the scalar potential $\phi$;

(4) the computation of electric field $\bar{E}$ using $\bar{A}$ and $\phi$

(5) the computation of magnetic field $\bar{B}$ using $\bar{A}$.

In this technique, the source is described only in terms of current density, and the field equations are expressed only in terms of current. The use of the Lorentz condition assures that the current continuity equation, which is not explicitly used in this technique, is satisfied.

The second approach, the so-called monopole technique, involves

(1) the specification of current density $\bar{J}$ (or charge density $\rho$ );

(2) the use of $\bar{J}$ (or $\rho$ ) and the continuity equation to find $\rho$ (or $\bar{J})$;

(3) the use of $\bar{J}$ to find $\bar{A}$ and $\rho$ to find $\phi$;

(4) the computation of electric field $\bar{E}$ using $\bar{A}$ and $\phi$

(5) the computation of magnetic field $\bar{B}$ using $\bar{A}$.

In this technique, the source is described in terms of both current density and charge density, and the field equations are expressed (a) in terms of charge density, or (b) in terms of current, or (c) in terms of both charge density and current. The current continuity equation is needed to relate the current density and charge density. There is no need for the explicit use of the Lorentz condition in this technique, although scalar and vector potentials do satisfy the Lorentz condition.

Rubinstein and Uman [1] used both the dipole and monopole techniques to derive expressions for electric fields for the case of a travelling step-function wave along a vertical antenna. Even though expressions for electric fields from the two techniques are very different in their structure, Rubinstein and Uman [1] were able to show numerically that they are equivalent. Later, Safaeinili and Mina [2] established the analytical equivalence between the two expressions for electric fields derived in [1]. In this paper, we extend these studies to an arbitrary source distribution on a vertical antenna and derive general expressions for electric field using the two approaches. These two expressions are analytically equivalent, eventhough different in form. We also show that the traditional division of electric field in the time domain into electrostatic, induction, and radiation components, based on these components' distance dependence, is not unique and depends upon the approach used.

Since the dipole technique uses Lorentz condition explicitly, it is called in this paper the Lorentz condition approach. Similarly, the monopole technique is called the continuity equation approach since it uses the continuity equation explicitly.

\section{THEORY AND ANALYSIS}

\section{The Lorentz Condition Approach}

The lightning return-stroke channel can be modeled as a straight line fixed at one end $\mathrm{A}$, with the other end extending with speed $v$. The geometry of the problem is shown in Fig. 1. The current on the lightning channel is represented by $i\left(z^{\prime}, t\right)$, where $z^{\prime}$ is the position along the $z$-axis with the origin at the base of the channel, and $t$ is the time. At time $t=0$ the return stroke starts to propagate from origin A. The observer at the fixed field point $P$ 'sees' the return stroke starting to propagate from the origin at time $t=r / c$, where $c$ is the speed of light. The retarded current at any elemental channel section $d z^{\prime}$ is given by $i\left(z^{\prime}, t-R\left(z^{\prime}\right) / c\right)$, where $z^{\prime}$ is less than or equal to $L^{\prime}(t)$, the length of the return stroke channel 'seen' by the observer at $P$ at time $t$. If the return-stroke wavefront moves at a constant speed $v$, then $L^{\prime}(t)$ is obtained from the solution of the equation $t=L^{\prime} / v+R(L) / c$. $L^{\prime}(t)$ is less than the actual length of the channel $v \cdot t$. Note that the assumption of constant return-stroke speed is not required in the derivations presented here. 
In fact, the lightning channel can be considered to be composed of many electric dipoles of length $d z^{\prime}$. Also, the current is assumed to be constant over the length of the dipole. Field expressions in the time domain, with specific application to lightning, are derived in [3], and later developed by $[1,4,5,6,7]$.

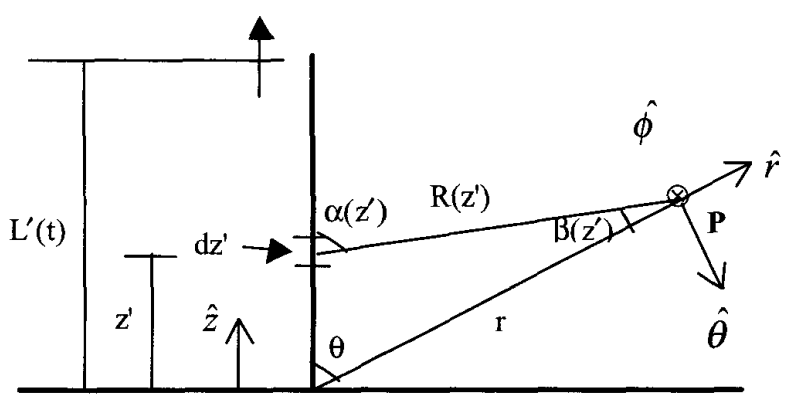

A

Figure 1. Geometry of the problem.

The vector potential at $P$ due to the entire extending channel is given by (equation 9 of [5])

$$
\bar{A}(r, \theta, \tau)=\frac{1}{4 \pi \varepsilon_{0} c^{2}} \int_{0}^{L^{\prime}(\tau)} \frac{i\left(z^{\prime}, \tau-R\left(z^{\prime} / c\right)\right.}{R\left(z^{\prime}\right)} \hat{z} d z^{\prime}
$$

where $\tau$ is a time less than or equal to time $t$. At time $\tau$, return-stroke wavefront is "seen" at a height $L^{\prime}$ ( $\left.\tau\right)$ by the observer at $P$ and $L^{\prime}(\tau)$ is less than or equal to $L^{\prime}(t)$. Note that in equation (1) we have not considered the presence of ground, usually assumed to be perfectly conducting and replaced by the channel image. The total electric field can be calculated using the relation

$$
\bar{E}=-\nabla \phi-\frac{\partial \bar{A}}{\partial t}
$$

where $\phi$ can be obtained from the Lorentz condition

$$
\begin{aligned}
& \nabla \bullet \bar{A}+\frac{1}{c^{2}} \frac{\partial \phi}{\partial t}=0, \text { as } \\
& \phi(r, \theta, t)=-c^{2} \int_{r / c}^{t} \nabla \bullet \bar{A} d \tau
\end{aligned}
$$

Taking the divergence of (1) it can be shown that

$$
\begin{aligned}
& \nabla \bullet \vec{A}(r, \theta, \tau)= \\
& +\frac{1}{4 \pi \varepsilon_{0} c^{2}} \int_{0}^{L^{\prime}(\tau)}\left[\begin{array}{c}
\frac{z^{\prime}-r \cos \theta}{R^{3}\left(z^{\prime}\right)} i\left(z^{\prime}, \tau-R\left(z^{\prime}\right) / c\right) \\
+\frac{z^{\prime}-r \cos \theta}{c R^{2}\left(z^{\prime}\right)} \frac{\partial i\left(z^{\prime}, \tau-R\left(z^{\prime}\right) / c\right)}{\partial \tau}
\end{array}\right] d z^{\prime} \\
& +\frac{1}{4 \pi \varepsilon_{0} c^{2}} \frac{L^{\prime}(\tau)-r \cos \theta}{c R^{2}\left(L^{\prime}\right)} i\left(L^{\prime}, \tau-R\left(L^{\prime}\right) / c\right) \frac{d L^{\prime}(\tau)}{d \tau}
\end{aligned}
$$

Substituting (4) into (3) and interchanging the order of integration, an expression for the scalar potential completely in terms of current can be obtained. As time increases from $r / c$ to $t$, the channel length $L^{\prime}(\tau)$ increases monotonically from 0 to $L^{\prime}(t)$. Therefore the order of integration can be changed as follows according to the standard rule.

$$
\int_{r / c}^{t} \int_{0}^{L^{\prime}(\tau)} \Rightarrow \int_{0}^{L^{\prime}(t)} \int_{\tau}^{t}
$$

where the lower limit $\tau=t_{b}$ is the time at which the observer at the field point 'sees' the return-stroke front at height $z$ ' for the first time. For a constant return-stroke speed $v$,

$$
\tau=\frac{L^{\prime}(\tau)}{v}+\frac{R\left(L^{\prime}(\tau)\right)}{c}=\frac{z^{\prime}}{v}+\frac{R\left(z^{\prime}\right)}{c}
$$

Performing the operations explained above and after some reductions, we get an expression for scalar potential as

$\phi(r, \theta, t)=$

$$
-\frac{1}{4 \pi \varepsilon_{0}} \int_{0}^{L^{\prime}(t)}\left[\begin{array}{l}
\frac{z^{\prime}-r \cos \theta}{R^{3}\left(z^{\prime}\right)} \int_{\frac{z^{\prime}}{v}+\frac{R\left(z^{\prime}\right)}{c}}^{t} i\left(z^{\prime}, t-R\left(z^{\prime}\right) / c\right) d \tau \\
+\frac{z^{\prime}-r \cos \theta}{c R^{2}\left(z^{\prime}\right)} i\left(z^{\prime}, t-R\left(z^{\prime}\right) / c\right)
\end{array}\right] d z^{\prime}
$$

Taking the gradient of equation (6), $\nabla \phi$ and the time derivative of equation (1), $\partial \bar{A} / \partial t$, we get an expression for electric field according to equation (2) as given below:

$$
\begin{aligned}
& \bar{E}(r, \theta, t)=-\frac{1}{4 \pi \varepsilon_{0}} \hat{r} \\
& \int_{0}^{L^{\prime}(t)}\left[\begin{array}{l}
\frac{\cos \theta-3 \cos \alpha\left(z^{\prime}\right) \cos \beta\left(z^{\prime}\right)}{R^{3}\left(z^{\prime}\right)} \int_{t_{b}}^{t} i\left(z^{\prime}, \tau-\frac{R\left(z^{\prime}\right)}{c}\right) d \tau \\
+\frac{\cos \theta-3 \cos \alpha\left(z^{\prime}\right) \cos \beta\left(z^{\prime}\right)}{c R^{2}\left(z^{\prime}\right)} i\left(z^{\prime}, t-\frac{R\left(z^{\prime}\right)}{c}\right) \\
+\frac{\cos \theta-\cos \alpha\left(z^{\prime}\right) \cos \beta\left(z^{\prime}\right)}{c^{2} R\left(z^{\prime}\right)} \frac{\partial i\left(z^{\prime}, t-R\left(z^{\prime}\right) / c\right)}{\partial t}
\end{array}\right] d z^{\prime} \\
& +\frac{1}{4 \pi \varepsilon_{0}} \hat{\theta} \\
& \int_{0}^{L^{\prime}(t)}\left[\begin{array}{l}
\frac{\sin \theta+3 \cos \alpha\left(z^{\prime}\right) \sin \beta\left(z^{\prime}\right)}{R^{3}\left(z^{\prime}\right)} \int_{t_{b}}^{t} i\left(z^{\prime}, \tau-\frac{R\left(z^{\prime}\right)}{c}\right) d \tau \\
+\frac{\sin \theta+3 \cos \alpha\left(z^{\prime}\right) \sin \beta\left(z^{\prime}\right)}{c R^{2}\left(z^{\prime}\right)} i\left(z^{\prime}, t-\frac{R\left(z^{\prime}\right)}{c}\right) \\
+\frac{\sin \theta+\cos \alpha\left(z^{\prime}\right) \sin \beta\left(z^{\prime}\right)}{c^{2} R\left(z^{\prime}\right)} \frac{\partial i\left(z^{\prime}, t-R\left(z^{\prime}\right) / c\right)}{\partial t}
\end{array}\right] d z^{\prime} \\
& -\frac{1}{4 \pi \varepsilon_{0}} \hat{r} \frac{\cos \theta-\cos \alpha\left(L^{\prime}\right) \cos \beta\left(L^{\prime}\right)}{c^{2} R\left(L^{\prime}\right)} i\left(L^{\prime}, t-\frac{R\left(L^{\prime}\right)}{c}\right) \frac{d L^{\prime}}{d t} \\
& +\frac{1}{4 \pi \varepsilon_{0}} \hat{\theta} \frac{\sin \theta+\cos \alpha\left(L^{\prime}\right) \sin \beta\left(L^{\prime}\right)}{c^{2} R\left(L^{\prime}\right)} i\left(L^{\prime}, t-\frac{R\left(L^{\prime}\right)}{c}\right) \frac{d L^{\prime}}{d t}
\end{aligned}
$$

In equation (7), $d L^{\prime} / d t$ is the speed of the current wavefront as 'seen' by the observer at $\mathrm{P}$, which is different from the real speed $v$. Also, from Fig. 1 we get $\cos \alpha\left(z^{\prime}\right)=-\left(z^{\prime}-r \cos \theta\right) / R\left(z^{\prime}\right), \cos \beta\left(z^{\prime}\right)=(r-$ 
$\left.z^{\prime} \cos \theta\right) / R\left(z^{\prime}\right)$, and $\sin \beta\left(z^{\prime}\right)=z^{\prime} \sin \theta / R\left(z^{\prime}\right)$. In the electric field expression (7) terms containing the factors $R^{-3}, c^{-1} R^{-2}$, and $c^{-2} R^{-1}$ are called the static component, the induction component, and the radiation component, respectively. The last two terms of the expression (7) containing $d L^{\prime} / d t$ will have non-zero values only if there is a current discontinuity (non-zero current) at the wavefront. The lower limit of the time integral of the first term in (7), $t_{b}$, is the time at which the return-stroke wavefront has reached the height $z^{\prime}$ for the first time, as 'seen' from the observation point.

A common problem in lightning is to find the electric and magnetic fields at ground level from a lightning return-stroke, whose channel is straight and vertical above ground. In this case $\theta=90^{\circ}$ and $\hat{\theta}=-\hat{z}$. The ground is assumed to be perfectly conducting. The effect of ground plane can be included by considering an image channel carrying an image current. The direction of this image current is the same as the current in the channel. $\hat{r}$-directed field components corresponding to the image channel are equal in magnitude but opposite in sign to the $\hat{r}$-directed components in (7). However, $\hat{\theta}$ directed components corresponding to the image channel are equal in both magnitude and sign to those in (7). Therefore adding the contribution of the image channel to (7), we get the complete field expression at ground $\left(\theta=90^{\circ}\right)$ as,

$$
\begin{aligned}
& E_{V}(r, t)=\frac{1}{2 \pi \varepsilon_{0}} \hat{z} \\
& {\left[\begin{array}{l}
\int_{0}^{L^{\prime}(t)} \frac{2-3 \sin ^{2} \alpha\left(z^{\prime}\right)}{R^{3}\left(z^{\prime}\right)} \int_{t_{b}}^{t} i\left(z^{\prime}, \tau-R\left(z^{\prime}\right) / c\right) d \tau d z^{\prime} \\
+\int_{0}^{L^{\prime}(t)} \frac{2-3 \sin ^{2} \alpha\left(z^{\prime}\right)}{c R^{2}\left(z^{\prime}\right)} i\left(z^{\prime}, t-R\left(z^{\prime}\right) / c\right) d z^{\prime} \\
-\int_{0}^{L^{\prime}(t)} \frac{\sin ^{2} \alpha\left(z^{\prime}\right)}{c^{2} R\left(z^{\prime}\right)} \frac{\partial i\left(z^{\prime}, t-R\left(z^{\prime}\right) / c\right)}{\partial t} d z^{\prime} \\
-\frac{\sin ^{2} \alpha\left(L^{\prime}\right)}{c^{2} R\left(L^{\prime}\right)} i\left(L^{\prime}, t-R\left(L^{\prime}\right) / c\right) \frac{d L^{\prime}}{d t}
\end{array}\right]}
\end{aligned}
$$

Individual terms on the right hand side of equation (8) are labeled the electrostatic, induction, and radiation components. It is customary to identify the electrostatic component by its $\mathrm{R}^{3}$ distance dependence, induction components by their $\mathrm{R}^{-2}$ dependence, and radiation components by $R^{1}$ dependence. The last term in equation (8) becomes zero if there is no current discontinuity at the propagating wavefront, i.e. if $i\left(L^{\prime}, t-R\left(L^{\prime}\right) / c\right)=0$.

\section{The Continuity Equation Approach}

The purpose here is to find an expression for electric field using both scalar potential and vector potential related by the continuity equation that defines the relationship between the charge density and current locally. The continuity equation that relates the charge density and current locally, but at retarded time is given in $[7,8]$ as

$$
\frac{\partial \rho^{*}\left(z^{\prime}, t-\frac{R\left(z^{\prime}\right)}{c}\right)}{\partial t}=-\left.\frac{\partial i\left(z^{\prime}, t-\frac{R\left(z^{\prime}\right)}{c}\right)}{\partial z^{\prime}}\right|_{t \frac{R\left(z^{\prime}\right)}{c}=\text { const } .}
$$

In equation (9), the partial differentiation of retarded current with respect to the source coordinate $z^{\prime}$ is carried out keeping the retarded time constant. That is, the dependence of $R\left(z^{\prime}\right)$ on $z^{\prime}$ is ignored while taking the partial derivative. Note that as viewed by an observer at a remote point $P$, the relationship between the charge density and current could be different from that given in (9) [4].

The returnstroke starts from the ground level $\left(z^{\prime}=0\right)$. To satisfy the continuity equation (9) at $z^{\prime}=0$, a point charge $Q(t-r / c)$ is required at $z^{\prime}=0$ as the source for the current emerging from $z^{\prime}=0$. This stationary point charge is given by,

$$
Q(t-r / c)=-\int_{r / c}^{t} i(0, \tau-r / c) d \tau
$$

The scalar potential from the whole lightning channel is given by

$$
\phi(r, t)=\frac{1}{4 \pi \varepsilon_{0}}\left[\frac{Q\left(t-\frac{r}{c}\right)}{r}+\int_{0}^{L^{\prime}(t)} \frac{\rho^{*}\left(z^{\prime}, t-\frac{R\left(z^{\prime}\right)}{c}\right)}{R\left(z^{\prime}\right)} d z^{\prime}\right]
$$

The electric field can be obtained from equation (2). Using the spherical coordinate system centered at the starting point of the return stroke at ground (Fig. 1) and ignoring the presence of ground for the moment, the negative gradient of the scalar potential $-\nabla \phi$ and the negative time derivative of the vector potential $-\partial A / \partial t$ can be found as described below. For $-\nabla \phi$ we have:

$$
\begin{aligned}
& -4 \pi \varepsilon_{0} \nabla \phi= \\
& -\hat{r} \frac{\partial}{\partial r} \frac{Q(t-r / c)}{r}-\hat{r} \frac{\partial}{\partial r} \int_{0}^{L^{\prime}(t)} \frac{\rho^{*}\left(z^{\prime}, t-R\left(z^{\prime}\right) / c\right)}{R\left(z^{\prime}\right)} d z^{\prime} \\
& -\hat{\theta} \frac{1}{r} \frac{\partial}{\partial \theta} \int_{0}^{L^{\prime}(t)} \frac{\rho^{*}\left(z^{\prime}, t-R\left(z^{\prime}\right) / c\right)}{R\left(z^{\prime}\right)} d z^{\prime}
\end{aligned}
$$

Note that the first term of equation (12) is independent of the spatial coordinate $\theta$. The maximum length of the channel $L^{\prime}(t)$, as seen from the field point, is a function of $r, \theta$, and $t$. The distance to the field point from the differential channel segment $R\left(z^{\prime}\right)$ is a function of both $r$ and $\theta$, as given by equations (13).

$$
\begin{aligned}
& R\left(z^{\prime}\right)=\sqrt{r^{2}+z^{\prime 2}-2 r z^{\prime} \cos \theta} \\
& \frac{d R\left(z^{\prime}\right)}{d r}=\frac{r-z^{\prime} \cos \theta}{R\left(z^{\prime}\right)} \\
& \frac{d R\left(z^{\prime}\right)}{d \theta}=\frac{r z^{\prime} \sin \theta}{R\left(z^{\prime}\right)}
\end{aligned}
$$

Carrying out the differentiation of the second and third terms in equation (12) and using equations (13), we obtain the following expression 


$$
\begin{aligned}
& -4 \pi \varepsilon_{0} \nabla \phi= \\
& +\hat{r} \int_{0}^{L^{\prime}(t)}\left[\begin{array}{l}
\frac{r-z^{\prime} \cos \theta}{R^{3}\left(z^{\prime}\right)} \rho^{*}\left(z^{\prime}, t-R\left(z^{\prime}\right) / c\right) \\
+\frac{r-z^{\prime} \cos \theta}{c R^{2}\left(z^{\prime}\right)} \frac{\partial \rho^{*}\left(z^{\prime}, t-R\left(z^{\prime}\right) / c\right)}{\partial t}
\end{array}\right] d z^{\prime} \\
& +\hat{\theta} \int_{0}^{L^{\prime}(t)}\left[\begin{array}{l}
\frac{z^{\prime} \sin \theta}{R^{3}\left(z^{\prime}\right)} \rho^{*}\left(z^{\prime}, t-R\left(z^{\prime}\right) / c\right) \\
+\frac{z^{\prime} \sin \theta}{c R^{2}\left(z^{\prime}\right)} \frac{\partial \rho^{*}\left(z^{\prime}, t-R\left(z^{\prime}\right) / c\right)}{\partial t}
\end{array}\right] d z^{\prime} \\
& -\hat{r} \frac{\rho^{*}\left(L^{\prime}, t-R\left(L^{\prime}\right) / c\right)}{R\left(L^{\prime}\right)} \frac{\partial L^{\prime}}{\partial r} \\
& -\hat{\theta} \frac{\rho^{*}\left(L^{\prime}, t-R\left(L^{\prime}\right) / c\right)}{r R\left(L^{\prime}\right)} \frac{\partial L^{\prime}}{\partial \theta}-\hat{r} \frac{\partial}{\partial r} \frac{Q(t-r / c)}{r}
\end{aligned}
$$

The time derivative of vector potential (1) is given by,

$$
\begin{aligned}
-4 \pi \varepsilon_{0} \frac{\partial \bar{A}}{\partial t}= & -\hat{z} \int_{0}^{L^{\prime}(t)} \frac{1}{c^{2} R\left(z^{\prime}\right)} \frac{\partial i\left(z^{\prime}, t-R\left(z^{\prime}\right) / c\right)}{\partial t} d z^{\prime} \\
& -\hat{z} \frac{i\left(L^{\prime}, t-R\left(L^{\prime}\right) / c\right)}{c^{2} R\left(L^{\prime}\right)} \frac{d L^{\prime}}{d t}
\end{aligned}
$$

The general expression for electric field at a field point can be found by combining equations (14), (15) and (16), as given below in (17).

We are interested in the return stroke field at ground level. For this case, $\theta=90^{\circ}$, and therefore $\cos \theta=0, \sin \theta=1$, and $\hat{\theta}=-\hat{z}$. The unit vector $\hat{r}$ is now horizontal, pointing away from the channel. A perfectly conducting plane at $z^{\prime}=0$ is introduced to simulate the effect of earth. Using the image theory, we can replace this plane by an image channel carrying current in the same direction as that in the actual channel. Writing equations for the image channel and adding them to equation (17) for the case of $\theta=90^{\circ}$, we get the expression for E-field, as follows.

$$
\begin{aligned}
& E_{V}(r, t)=-\frac{1}{2 \pi \varepsilon_{0}} \hat{z} \\
& {\left[\begin{array}{l}
\int_{0}^{L^{\prime}(t)} \frac{z^{\prime}}{R^{3}\left(z^{\prime}\right)} \rho^{*}\left(z^{\prime}, t-R\left(z^{\prime}\right) / c\right) d z^{\prime} \\
+\int_{0}^{L^{\prime}(t)} \frac{z^{\prime}}{c R^{2}\left(z^{\prime}\right)} \frac{\partial \rho^{*}\left(z^{\prime}, t-R\left(z^{\prime}\right) / c\right)}{\partial t} d z^{\prime} \\
+\int_{0}^{L^{\prime}(t)} \frac{1}{c^{2} R\left(z^{\prime}\right)} \frac{\partial i\left(z^{\prime}, t-R\left(z^{\prime}\right) / c\right)}{\partial t} d z^{\prime} \\
+\frac{L^{\prime}(t)}{c R^{2}\left(L^{\prime}\right)} \rho^{*}\left(L^{\prime}, t-R\left(L^{\prime}\right) / c\right) \frac{d L^{\prime}}{d t} \\
+\frac{1}{c^{2} R\left(L^{\prime}\right)} i\left(L^{\prime}, t-R\left(L^{\prime}\right) / c\right) \frac{d L^{\prime}}{d t}
\end{array}\right.}
\end{aligned}
$$

Note that by definition, the current and charge density in (18) are related by the local continuity equation (9). Equation (18) contains both current and charge density, while equation (8) contains only current. The first three terms of equation (18) are similar to the corresponding terms of the expression for E-field derived in the book of Jefimenko [9] for a volume charge and current distribution whose boundary is fixed in space.

$$
\begin{aligned}
& \bar{E}(r, \theta, t)=\frac{1}{4 \pi \varepsilon_{0}} \hat{r} \int_{0}^{L^{\prime}(t)}\left[\begin{array}{l}
-\frac{r-z^{\prime} \cos \theta}{R^{3}\left(z^{\prime}\right)} \rho^{*} \cdot\left(z^{\prime}, t-R\left(z^{\prime}\right) / c\right) \\
-\frac{r-z^{\prime} \cos \theta}{c R^{2}\left(z^{\prime}\right)} \frac{\partial \rho^{*}\left(z^{\prime}, t-R\left(z^{\prime}\right) / c\right)}{\partial t} \\
+\frac{\cos \theta}{c^{2} R\left(z^{\prime}\right)} \frac{\partial i\left(z^{\prime}, t-R\left(z^{\prime}\right) / c\right)}{\partial t}
\end{array}\right] d z^{\prime}+\frac{1}{4 \pi \varepsilon_{0}} \hat{\theta} \int_{0}^{L^{\prime}(t)}\left[\begin{array}{l}
-\frac{z^{\prime} \sin \theta}{R^{3}\left(z^{\prime}\right)} \rho^{*}\left(z^{\prime}, t-R\left(z^{\prime}\right) / c\right) \\
\left.-\frac{z^{\prime} \sin \theta}{c R^{2}\left(z^{\prime}\right)} \frac{\partial \rho^{*}\left(z^{\prime}, t-R\left(z^{\prime}\right) / c\right)}{\partial t}\right] d z^{\prime} \\
-\frac{\sin \theta}{c^{2} R\left(z^{\prime}\right)} \frac{\partial i\left(z^{\prime}, t-R\left(z^{\prime}\right) / c\right)}{\partial t}
\end{array}\right] \\
&-\frac{1}{4 \pi \varepsilon_{0}} \hat{r} \frac{r-L^{\prime} \cos \theta}{c R^{2}\left(L^{\prime}\right)} \rho^{*}\left(L^{\prime}, t-R\left(L^{\prime}\right) / c\right) \frac{d L^{\prime}(t)}{d t}+\frac{1}{4 \pi \varepsilon_{0}} \hat{r} \frac{\cos \theta}{c^{2} R\left(L^{\prime}\right)} i\left(L^{\prime}, t-R\left(L^{\prime}\right) / c\right) \frac{d L^{\prime}(t)}{d t} \\
&-\frac{1}{4 \pi \varepsilon_{0}} \hat{\theta} \frac{L^{\prime} \sin \theta}{c R^{2}\left(L^{\prime}\right)} \rho^{*}\left(L^{\prime}, t-R\left(L^{\prime}\right) / c\right) \frac{d L^{\prime}(t)}{d t}-\frac{1}{4 \pi \varepsilon_{0}} \hat{\theta} \frac{\sin \theta}{c^{2} R\left(L^{\prime}\right)} i\left(L^{\prime}, t-R\left(L^{\prime}\right) / c\right) \frac{d L^{\prime}(t)}{d t} \\
&+\frac{1}{4 \pi \varepsilon_{0}} \hat{r}\left[\frac{1}{r^{2}} \int_{r^{\prime} / c}^{t} i(0, \tau-r / c) d \tau+\frac{1}{r c} i(0, t-r / c)\right]
\end{aligned}
$$


By analogy with equation (8), the first term of equation (18) can be considered as representing the electrostatic field $\left(R^{-3}\right.$ dependence), the sum of second and fourth terms as representing the induction field ( $c^{-1} R^{-2}$ dependence), and the sum of third and last terms as representing the radiation field $\left(c^{-2} R^{-1}\right.$ dependence). It appears that the electrostatic, induction, and radiation terms (except for the last two terms associated with the wavefront) in (18) can also be identified as containing $z^{\prime}$ times line charge density (charge), $z^{\prime}$ times time derivative of line charge density (time derivative of charge) or current, and derivative of current, respectively. If there is no current or charge discontinuity at the wavefront, the last two terms become zero.

The magnetic field is given by $\bar{B}=\nabla \times \bar{A}$. The magnetic field expressions are identical in both approaches since it is completely determined by the vector potential given by equation (1). The expression for magnetic field at ground is $[3,4]$,

$$
\begin{aligned}
& B(r, t)=\frac{1}{2 \pi \varepsilon_{0} c^{2}} \hat{\phi} \\
& \int_{0}^{L^{\prime}(t)}\left[\begin{array}{l}
\frac{\sin \alpha\left(z^{\prime}\right)}{R^{2}\left(z^{\prime}\right)} i\left(z^{\prime}, t-R\left(z^{\prime}\right) / c\right) \\
+\frac{\sin \alpha\left(z^{\prime}\right)}{c R\left(z^{\prime}\right)} \frac{\partial i\left(z^{\prime}, t-R\left(z^{\prime}\right) / c\right)}{\partial t}
\end{array}\right] d z^{\prime} \\
& +\frac{1}{2 \pi \varepsilon_{0} c^{2}} \hat{\phi} \frac{\sin \alpha\left(L^{\prime}\right)}{c R\left(L^{\prime}\right)} i\left(L^{\prime}, t-R\left(L^{\prime}\right) / c\right) \frac{d L^{\prime}(t)}{d t}
\end{aligned}
$$

\section{Non-Uniqueness of Field Components}

Fields calculated using the Lorentz condition approach and the continuity equation approach, given by equation (8) and (18), respectively, should be identical since both are derived by rigorous application of electromagnetic principles and use the same basic assumptions. However the expressions look different in their structure. Therefore it would be interesting to compare the fields from (8) and (18) due to a known current distribution. It will be shown that while the total fields given by equations (8) and (18) are identical, the individual field components (electrostatic, induction, and radiation terms identified by their dependence on $R$ ) in these two equations are different. Take the following numerical example. Imagine the return stroke as a current wave that starts from the ground, and travels up with a constant speed $v$. In that case the current at any height $z^{\prime}$ at time $t$ is equal to the current at ground at an earlier time $t-z^{\prime} / v$, where $z^{\prime} / v$ is the travel time between ground and the height $z^{\prime}$. This is the so-called transmission line (TL) model and the current at any height is related to the current at ground by the following expression

$$
i\left(z^{\prime}, t\right)=i\left(0, t-z^{\prime} / v\right)
$$

Since there is no discontinuity at the wavefront for the TL model, the last term of equation (8) and the last two terms of equation (18) drop out of the equations. The charge density in equation (18) is calculated using the local continuity equation (9), which for the transmission line model can be rewritten as [4] $\rho^{*}\left(z^{\prime}, t-R\left(z^{\prime}\right) / c\right)=\frac{i\left(0, t-z^{\prime} / v-R\left(z^{\prime}\right) / c\right)}{v}$

where $v$ is the return stroke speed.

For a typical subsequent return stroke current waveform [10], computed electric fields at distances $50 \mathrm{~m}, 1 \mathrm{~km}$, and $100 \mathrm{~km}$ are shown in Figs. 2, 3, and 4, respectively. In the curve labels in Figures 2, 3, and 4, LC indicates the terms in equation (8), and CE indicates the terms in equation (18). The labels EQ, EI, and ER indicate the electrostatic $\left(\mathrm{R}^{-3}\right.$ dependence), induction $\left(\mathrm{c}^{-1} \mathrm{R}^{-2}\right.$ dependence), and radiation $\left(\mathrm{c}^{-2} \mathrm{R}^{-1}\right.$ dependence) field components. The following can be observed from Figs. 2, 3, and 4 and from equations (8) and (18).

1. The total fields given by equations (8) and (18) are identical (for up to several decimal places when numbers are compared).

2. In equation (18), the electrostatic and induction terms are given completely by the gradient of the scalar potential, while the radiation term is completely given by the time derivative of the vector potential. In contrast, in equation (8), both the gradient of the scalar potential and the time derivative of the vector potential contribute to the radiation field term.

3. The electrostatic $\left(\mathrm{R}^{-3}\right.$ dependence), induction $\left(\mathrm{c}^{-1} \mathrm{R}^{-2}\right.$ dependence), and radiation $\left(c^{-2} R^{-1}\right.$ dependence) terms in (8) are different from the corresponding terms in (18). The difference is considerable at $50 \mathrm{~m}$ (very close to the channel) and almost negligible at $100 \mathrm{~km}$ (far away from the channel).

4. At $50 \mathrm{~m}$ (very close to the channel), the electrostatic term $\left(\mathrm{R}^{-3}\right.$ dependence) in equation (8) is larger than its counterpart in equation (18) (compare curves EQ_LC and EQ_CE in Fig. 2).

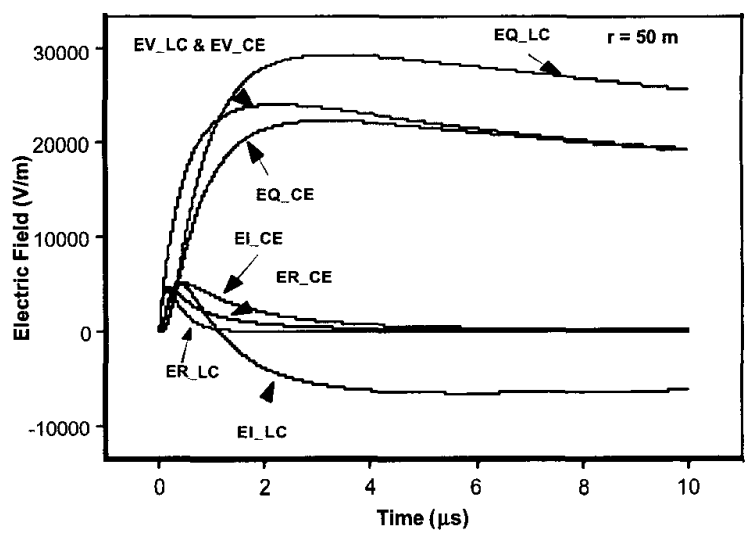

Figure 2. Comparison of the total electric field and its components at a distance of $50 \mathrm{~m}$ predicted by the TL model and field expressions (8) and (18). "LC" (Lorentz condition) at the end of the label corresponds to equation (8), and "CE" (Continuity equation) to equation (18).

The above analysis clearly shows that, even though the total electric field from a current or charge distribution is unique, the division of total electric field in the time domain into so-called electrostatic $\left(R^{-3}\right.$ dependence), induction $\left(c^{-1} R^{-2}\right.$ dependence), and radiation $\left(c^{-2} R^{-1}\right.$ dependence) components is not unique. This was further verified by calculating the individual field components and the total fields using 
six different return stroke models (BG, TCS, MTLE, MTLL, and DU models described in [10]). Of these models, the BG and TCS models have current discontinuity at the wavefront whereas other models do not have wavefront current discontinuity.

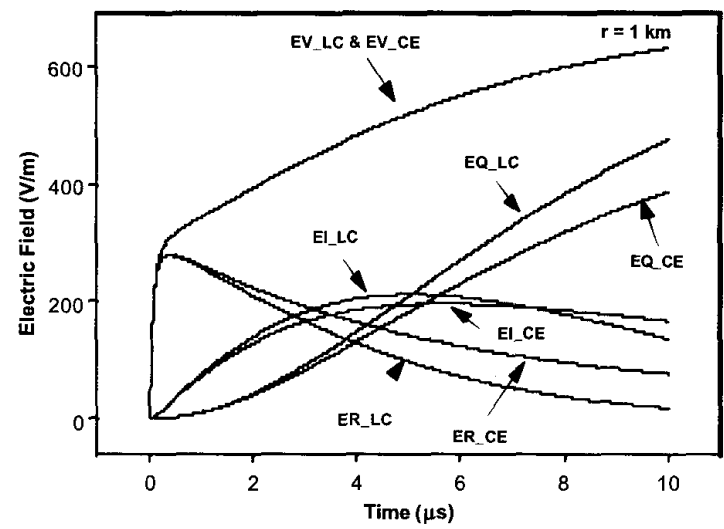

Figure 3. Same as Fig. 2, but at a distance of $1 \mathrm{~km}$.

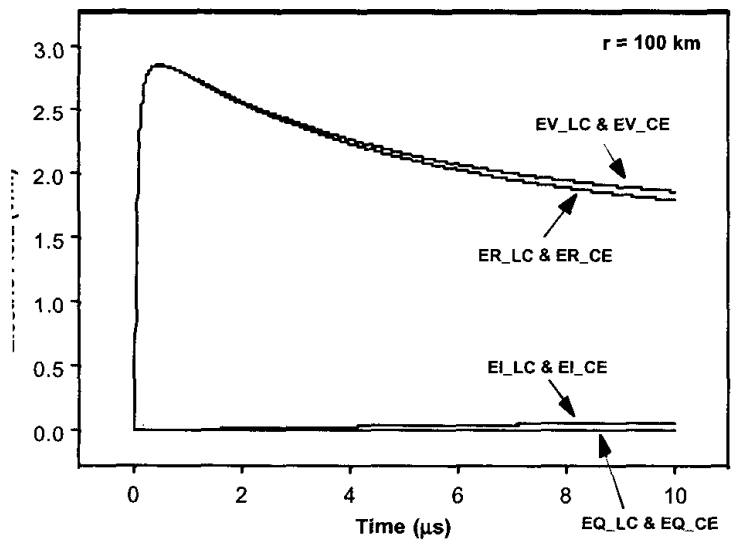

Figure 4. Same as Fig. 2, but at a distance of $100 \mathrm{~km}$.

Note that in the Lorentz condition technique all field components are expressed in terms of current, while in the continuity equation technique both current and charge density are involved. In the Lorentz condition technique the gradient of scalar potential contributes to all the three field components, whereas in the continuity equation technique, it contributes only to the electrostatic and induction field components. In either case, the expression for magnetic field at ground level is the same, equation (19), since it depends only on the vector potential. We get the same Poynting vector whether we calculate it from equation pairs (8) and (19) or (18) and (19), since the total electric fields given by (8) and (18) are the same. In fact equation (8) can be analytically derived from equation (18).

\section{SUMMARY}

General expressions for electric field due to an arbitrary source distribution on a vertical lightning channel, modeled as a linear antenna, are derived using two different approaches, the Lorentz condition (or dipole) approach and the continuity equation (or monopole) approach. It is shown that both approaches give the same total field, but the individual field components, static, induction, and radiation, as identified by their distance dependence, are not unique. The differences between field components are significant at close distances and negligible at far distances.

\section{REFERENCES}

[1] Rubinstein, M., and M.A. Uman, Methods for calculating the electromagnetic fields from a known source distribution: Application to lightning, IEEE Trans. Electromagn. Comp., 31, 183-189, 1989.

[2] Safaeinili, A., and M. Mina, On the analytical equivalence of electromagnetic fields solutions from a known source distribution, IEEE Trans. Electromagn. Comp., 33, 69-71, 1991.

[3] Uman, M. A., D. K. McLain, and E. P. Krider, The electromagnetic radiation from a finite antenna, Am. J. Phys., 43, 33$38,1975$.

[4] Thottappillil, R., V.A. Rakov, and M.A. Uman, Distribution of charge along the lightning channel: Relation to remote electric and magnetic fields and to return-stroke models, J. Geophys. Res., 102, 6987-7006, 1997.

[5] Thottappillil, R., M. A. Uman, and V. A. Rakov, Treatment of retardation effects in calculating the radiated electromagnetic fields from the lightning discharge, J. Geophys. Res., 103, 9003-9013, 1998.

[6] Rubinstein, M. and M. A. Uman, On the radiation field turn-on term associated with travelling current discontinuities in lightning, J. Geophys. Res., 95, 3711-3713, 1990.

[7] Thomson, E.M., Exact expressions for electric and magnetic fields from a propagating lightning channel with arbitrary orientation. J. Geophys. Res., 104, 22,293-22,300, 1999.

[8] Stratton, Electromagnetic theory, McGraw-Hill, Inc., 1941

[9] Jefimenko, O.D., Electricity and magnetism, Second edition, Electret Scientific Company, Star City, USA, 1989.

[10] Nucci, C. A., G. Diendorfer, M. A. Uman, F. Rachidi, M. Ianoz, and C. Mazzetti, Lightning return stroke current models with specified channel-base current: A review and comparison, J. Geophys. Res., 95, 20395-20408, 1990.

[11] Rakov, V.A., and Uman, M.A., Review and evaluation of lightning return stroke models including some aspects of their application. IEEE Trans. Electromagn. Comp., 40, 403-426, 1998. 